\title{
Medium-term Survival Following of Stroke in Yaoundé (Cameroon): a prospective observational cohort study
}

\section{Guy Sadeu Wafeu ( $\square$ wafeuguy@yahoo.fr)}

Faculty of Medicine and Biomedical Sciences https://orcid.org/0000-0002-6192-9580

\section{Leo Fozeu}

Faculty of Medecine and Biomedical Science

Pierre Ongolo-zogo

Faculty of Medicine and Biomedical Sciences

\section{Alain Patrick Menanga}

Faculty of Medicine and Biomedical Sciences

\section{Alfred Kongnyu Njamnshi}

Faculty of Medecine and Biomedical Sciences

\section{Research article}

Keywords: Stroke, prognosis factors, mortality, survival, Cameroon

Posted Date: October 8th, 2019

DOI: https://doi.org/10.21203/rs.2.15742/v1

License: (c) (i) This work is licensed under a Creative Commons Attribution 4.0 International License. Read Full License

Version of Record: A version of this preprint was published at Journal of Stroke and Cerebrovascular Diseases on September 1st, 2020. See the published version at https://doi.org/10.1016/j.jstrokecerebrovasdis.2020.105060. 


\section{Abstract}

With 5.7 million deaths per year, stroke is the second cause of mortality worldwide, and $70 \%$ of these deaths occur in developing countries especially in relation to inappropriate clinical pathways and resources. The aim of our study was to assess the survival rate of stroke patients within 90 days and to identify its determinants. It was a prospective observational cohort study over a period of 90 days after stroke. Patients were recruited between February and May 2015 in two tertiary hospitals in Yaoundé. The mortality rate was obtained by the Kaplan-Meier method. Multivariate analysis was performed using a Cox proportional hazards model. Sixty-six patients were enrolled of which 54 were followed up to 90 days. The overall mortality rate was $23.2 \%$ (95\% Cl: $12.5-87.5)$, more than two-thirds of the deaths occurred within the first 30 days. The mortality rates at days 14, 30, 60 day were $9.1 \%$ (95\% Cl: $3.0-$ 16.7), $14.3 \%$ (95\% Cl: 6.3 - 23.8) and $21.1 \%$ (95\% Cl: 10.5 - 31.6) respectively. High systolic blood pressure and a low Glasgow coma score on admission were independent risk factors of mortality at 90 days. The stroke related mortality compels appropriate collective mobilization for an early and adequate management of stroke patients.

\section{Background}

About 15 million people worldwide are affected by stroke each year. Over the last four decades, its incidence has increased by $100 \%$ in low/middle-income countries. In 2008 , for the first time, its incidence was higher than that in developed countries [1]. With 5.7 million deaths a year, it is the second leading cause of death in the world after ischemic heart disease [2]. More than $70 \%$ of these deaths occur in developing countries [1]. In sub-Saharan Africa, hospital-based studies have found stroke to be the leading cause of admission in neurology, with a mortality during admission going up to $43 \%$ in some cases [3,4]. In Cameroon, despite a cost of treatment averaging 1382USD (22 times the minimal monthly wage) per patient [5], intra-hospital stroke mortality remains high. However, the stroke medium-term survival and its determinants have sparsely studied in sub Saharan countries. The aim of our study was to assess the survival rate of stroke patients within 90 days, and to investigate the determinants of stroke-related mortality.

\section{Patients And Methods}

Yaoundé is the political capital of Cameroon, with about 1.5 million inhabitants. Our study took place in two out of five tertiary hospitals with permanent neurologists and cardiologists, namely the Central Hospital and the General Hospital. Most stroke patients in the city are referred to these facilities. This was a prospective observational cohort study lasting 90 days after the stroke. Patients were recruited from February $1^{\text {st }}$ until May $30^{\text {th }}, 2015$. We included all adult patients received for a sudden onset of neurological deficit with a brain computerized tomography (CT) scan suggestive of cerebral infarction or intra cerebral haemorrhage. We excluded any patient who did not have a brain CT scan or whose scan featured other findings such as meningeal haemorrhage and cerebral venous thrombosis. Our sampling method was consecutive and exhaustive. 
Data were collected using a pre-established data sheet and patients or relatives were contacted through phone call on five occasions. The first contact was made at the patient's bed within 24 hours of admission. During this contact, the following information was obtained from the patient or his entourage: demographic data (age, sex, marital status, level of education, dominant side), the recruitment hospital and service where the patient was admitted, the patient medical history (high blood pressure, diabetes, dyslipidaemia, smoking, alcoholism, history of stroke and the duration between that and the current episode, history of embolic heart disease, human immunodeficiency virus infection or sickle cell disease), the time in hours between the onset of symptoms and arrival at a health facility, the time taken to arrive at the recruitment hospital and the delay in performing the brain CT scan. For patients whose symptoms were noticed on waking, we considered that they started at bedtime. The patient was then examined to evaluate the Glasgow coma score and the national institutes of health stroke scale (NIHSS).

The summary of the brain CT report provides details the type of stroke, the presence or absence of mass effect, the ischemic territory, the site for intra-cerebral haemorrhages, the presence or absence of ventricular or meningeal haemorrhage, presence or absence of brain herniation and its topography, the volume of the hematoma calculated using the formula developed by Kothari et al. [6]: volume $\left(\mathrm{mm}^{3}\right)=(A$ $\left.{ }^{*} B{ }^{*} C\right) / 2$, where $A$ is the largest diameter in centimetres $(\mathrm{cm})$ of the hematoma on the scan, $B$ is the diameter perpendicular to $A$ and $C$ is the thickness on which the hematoma is visible (number of CT slices $x$ slice thickness). The results of biological workups were noted, e.g.; random or fasting blood sugar, total cholesterol, low-density lipoprotein (LDL) cholesterol, high-density lipoprotein (HDL) cholesterol and triglycerides, blood urea level, serum creatinin, full blood count, human immunodeficiency virus infection (HIV) and syphilis serologies, transaminases (aspartate aminotransferase (AST) and alanine aminotransferase (ALT)), ABO and Rhesus blood grouping. We also collected the results of electrocardiogram (presence or absence of atrial fibrillation) and ultrasound of supra-aortic arteries (mostly percentage of arterial stenosis).

The 04 (four) other contacts were on the 14th, 30th, 60th and 90th days after the stroke. Patients or their relatives were contacted by phone to find out if the patient was alive or not. For deceased patients, the day of death was recorded, so as to calculate survival time after stroke.

\section{Statistical Analysis}

Data entry and analysis was done using SPSS for windows version 20. Survival probabilities were obtained by the Kaplan Meier method. The difference between survival curves was determined using the Log Rank test. The effect of variables that were significantly associated with prognosis was then studied in multivariate analysis using a Cox proportional hazards model. Data were analysed using SPSS version 20 (SPSS Inc, Chicago, IL). A p value $<0.05$ was considered statistically significant.

Ethical considerations: Prior to the start of the study, research authorizations were obtained from the directors of the various hospitals. The research protocol was submitted for approval by the Institutional Ethics and Research Committee of the Faculty of Medicine and Biomedical Sciences of the University of 
Yaoundé I. Included in the study were patients who freely consented to participate, after explanation of the advantages and disadvantages. For unconscious patients, consent was obtained from family members.

\section{Results}

Sixty six patients were recruited, 48 at Central Hospital and 18 at General Hospital. All patients were contacted on day 14 , sixty three on day 30 , fifty seven on day 60 and fifty six on day 90 . The mean age was $59.2 \pm 13.7$ years (the extremes being 26 and 85 years). The sex ratio was 1 . The other sociodemographic characteristics are described in Table 1.

The most common cerebrovascular risk factor was arterial hypertension, present in 41 (62.1\%) patients; 8 (12.1\%) patients were known to be diabetic, $7(10.6 \%)$ had a history of confirmed stroke, the median time between the last and the current was $30(12-48)$ months, with extremes 5 and 48 months. Two patients had an embolic heart disease.

Thirteen patients $(19.7 \%)$ were smokers, of whom 8 (61.5\%) were former smokers. The smoking index ranged between 5 and 45 pack years (PY), with a median of 17.5 (7.3 - 30) PY. Forty-four patients (66.6\%) were alcohol drinkers, of whom 8 (18.9\%) were former consumers. The median alcohol index was 6 (4 57) $\mathrm{g}$ with extremes of 1 and $114 \mathrm{~g}$. Fifteen patients (22.7\%) had no known cerebrovascular risk factor before the stroke.

Regarding the consultation time, $12(18.2 \%)$ patients consulted at one of the recruiting hospitals within 3 hours of onset of symptoms and $16(24.2 \%)$ within 6 hours. Two patients did the brain scan within 6 hours following the onset of symptoms. On admission, 30 (45.4\%) patients had a Glasgow coma score less than 15 , of whom $3(4.5 \%)$ had a score less than 9 . Ten patients $(15.2 \%)$ had dysphagia and 22 patients $(33.3 \%)$ had detrusor-external sphincter dyssynergia. Five patients $(7.5 \%)$ had seizures before consultation. Table 2 gives the details of the different times and parameters at admission.

On CT, cerebral infarction was found in 35 patients (53\%) while intracerebral hematoma was found in 31 patients $(47 \%)$. Lesions were predominantly on the left side $(59.1 \%$ of patients), with 2 patients (3\%) having bilateral lesions. Sixteen patients $(24.2 \%)$ had a mass effect on the medial structures, with a subfalcine herniation in six of them (9.1\%). The median volume of the hematoma was 9.2 (2.9-14.7) $\mathrm{ml}$. Table 3 presents the topographic features of the different types of stroke.

For the patients who performed the biological tests, there was $20 \%(9 / 44)$ with a blood glucose higher than $1.4 \mathrm{~g} / \mathrm{l}$. Serum creatinine was elevated in 22 patients (32.2\%), a hyperuricemia found in 6 of the 14 patients in whom the serum uric acid was measured. $60.5 \%$ of patients $(26 / 43)$ had a lipid disorder, the majority of whom had elevated LDL cholesterol. There was anaemia in 17 patients (28.8\%), thrombocytopenia in the same number and leucocytosis in 19 patients (32.2\%). 
A total of 13 patients died, giving a 90 -day overall mortality rate of $23.2 \%$ (95\% Cl: $12.5-87.5)$. Mortality at Day 14, Day 30 and Day 60 were 9.1\% (95\% Cl: 3.0-16.7), 14.3\% (95\% Cl: 6.3-23.8) and 21.1\% (95\% Cl: 10.5-31.6), respectively. Figure 1 shows the survival curve of the patients.

After the univariate analysis, the variables associated with the risk of death were age greater than 58 years $(p=0.026)$, the presence of dysphagia on admission $(p<0.001)$, the presence of detrusor-external sphincter dyssynergia $(p<0.001)$, admission parameters including Glasgow coma score $(p<0.001)$, systolic blood pressure $(p=0.03)$, respiratory rate $(p=0.006)$, temperature $(p=0.001)$ and the NIHSS $(p=$ 0.004). In patients with cerebral hematoma, the presence of ventricular flood was associated with the risk of death $(p<0.001)$. Figure 2 shows the survival curves according to the presence or absence of a ventricular flood. At the end of the multivariate analysis including these variables, the Glasgow coma score and systolic blood pressure on admission appeared as independent 90-day mortality factors. Table IV shows the results of the multivariate analysis.

\section{Discussion}

The objective of our study was to assess the survival rate of stroke patients, and the factors associated with Medium-term Survival. Overall mortality at 90 days post-stroke was $23.2 \%$. It is close to the $26.8 \%$ intra-hospital mortality found by Mapouré et al. in 2014 at the Douala General Hospital [7] in almost identical socio-economic environment. This mortality is lower than the $41 \%$ and $35 \%$ found by Garbusinski et al. in The Gambia in 2001 and Ekeh et al. in Nigeria in 2006 respectively [8,9]. This could be explained by the improvement in the quality of care in sub-Saharan Africa over the last ten years with the increase in number of specialists. However, this mortality remains higher than in developed countries. Furthermore, approximately $70 \%$ of deaths occurred within the first 30 days after stroke. It is therefore important to reinforce the quality of the care of stroke patients in our context, especially in the early phase of the disease.

The Glasgow coma score was a factor independently associated with mortality. This result is similar to that of most studies on the prognosis of stroke in sub-Saharan Africa [7-10]. An altered level of consciousness reflects the severity of the pathology and exposes the patient to more infectious complications particularly through swallowing disorders and detrusor-external sphincter dyssynergia. Access to resuscitation services in our context is not guaranteed, because of the high cost. Systolic hypertension was also an independent factor associated with mortality. This result has not been found in other studies. It could be explained by the fact that recommendations on the management of hypertension in the acute phase of stroke are not strictly followed, thus increasing mortality in patients with high systolic blood pressure.

The type of stroke had no significant association with mortality, unlike the results of Mapoure et al. in 2014 [7]. Indeed, in our study population, the median volume of hematoma was low $(9.2 \mathrm{ml})$ compared to other studies. This volume is a severity factor of cerebral hematomas, and has been described as a predictor of mortality when greater than $60 \mathrm{ml}$ [11]. Nonetheless, the presence of ventricular flood was 
significantly associated with mortality in the haemorrhagic stroke group. Hyperglycaemia on admission was found to be an independent predictor of intra-hospital mortality in Douala [7]. But we did not measure blood sugar level on admission in our study. However, our study has some limitations, including the fact that it only concerns patients who have had a brain CT scan. It is an hospital-based study and does not take into account patients who died before arriving at the hospital. Biological workups were not performed in the same laboratory and management was not standardized for all patients.

\section{Conclusion}

Medium-term survival following stroke remains low in our context, with $70 \%$ of deaths occurring within the first 30 days. Altered consciousness and systolic hypertension on admission are independent factors associated with mortality. It is therefore important to reinforce the quality of the care of stroke patients in our context, especially in the early phase of the disease and for unconscious patients.

\section{List Of Abbreviations}

ACA: Anterior Cerebral Artery

ALT: Alanine aminotransferase

AST: Aspartate aminotransferase

CT: Computerized Tomography

ECG: Electrocardiogram

HDL: High Density Lipoprotein

HIV: Human Immunodeficiency Virus

LDL: Low Density Lipoprotein

MCA: Middle Cerebral Artery

NIHSS: National Institutes of Health Stroke Scale

PY: Packs Year

USD: United States Dollar

\section{Declarations}

\section{Ethics approval and consent to participate}


Ethics approval was obtain from the Institutional Ethics and Research Committee of the Faculty of Medicine and Biomedical Sciences of the University of Yaoundé I. Research authorizations were obtained from the directors of the various hospitals. We included in the study patients who freely consented to participate and signed the consent form. For unconscious patients, consent was obtained from family members.

\section{Consent for publication}

Not Applicable

\section{Availability of data and materials}

The datasets used and/or analysed during the current study are available from the corresponding author on reasonable request.

\section{Competing interests}

The authors declare that they have no competing interests.

\section{Funding}

None

\section{Authors' contributions}

GSW, POZ, APM and AKN conceived and design the study, and GSW collected data and follow-up the patients. GSW and LF performed statistical analysis and draft the manuscript. POZ, APM and AKN supported for the critical revision of the manuscript for intellectual content. All authors read and approved the final manuscript.

\section{Acknowledgments}

None

\section{References}

1. Sagui E. Les accidents vasculaires cérébraux en Afrique subsaharienne. Med Trop. 2007;67:596600 . 
2. The global burden of disease: 2004 Update [Internet]. Geneva: World Health Organisation; 2008 [cited 2014 Nov 13]. Available from:

www.who.int/healthinfo/global_burden_disease/GBD_report_2004update_full.pdf

3. Cowppli-Bony P, Sonan-Douayoua T, Akani F, Datie A-M, Assi B, Aka-Diarra E, et al. Epidémiologie des patients hospitalisés en neurologie: expérience du centre hospitalier universitaire de Cocody à Abidjan (Côte-d'Ivoire). AJNS. 2004;23(2):16-23.

4. Chapp-Jumbo EN. Neurologic admissions in the Niger delta area of Nigeria - a ten years review. AJNS. 2004;23(1):14-20.

5. Mapoure YN, Kuate C, Bibaya Anaba Kouna PE, Luma HN, Mouelle AS, Njamnshi AK. Coût des Accidents Vasculaires Cérébraux à l'Hôpital Général De Douala. Health Sci Dis. 2014 Sep;15(3):1-7.

6. Kothari RU, Brott T, Broderick JP, Barsan WG, Sauerbeck LR, Zuccarello M, et al. The ABCs of measuring intracerebral hemorrhage volumes. Stroke. 1996;27(8):1304-5.

7. Mapoure NY, Tchaleu Nguenkam CB, Mbatchou Ngahane HB, Dzudie A, Coulibaly A, Mounjouopou NG, et al. Predictors of In-Hospital Mortality for Stroke in Douala, Cameroon. Stroke Res Treat. 2014;2014:1-6.

8. Ekeh B, Ogunniyi A, Isamade E, Ekrikpo U. Stroke mortality and its predictors in a Nigerian teaching hospital. Afr Health Sci. 2015;15(1):74.

9. Garbusinski JM, van der Sande MAB, Bartholome EJ, Dramaix M, Gaye A, Coleman R, et al. Stroke presentation and outcome in developing countries: a prospective study in the Gambia. Stroke. 2005;36:1388-93.

10. Touré K, Diagne SN, Seck LB, Sow A, Ndiaye Moustapha, Diop MS, et al. Facteurs prédictifs de mortalité par accident vasculaire cérébral (AVC) à la clinique neurologique du CHU de Fann, DakarSénégal. Afr J Neurol Sci. 2010;29(2):29-36.

11. Broderick JP, Brott T, Duldner JE, Tomsick T, Huster G. Volume of intracerebral hemorrhage a powerful and easy-to-use predictor of 30-day mortality. Stroke. 1993;24(7):987-93.

\section{Tables}

Table 1: Socio-demographic characteristics of the study population 


\begin{tabular}{lll}
\hline Variables & Number & Percentage \\
\hline Age & 2 & 3 \\
[20-30[ & 2 & 3 \\
[30-40[ & 12 & 18.2 \\
[40-50[ & 19 & 28.8 \\
[50-60[ & 14 & 21.2 \\
[60-70[ & 12 & 18.2 \\
[70-80[ & 5 & 7.6 \\
[80-90[ & 16 & 24.2 \\
Level of education & \\
None & 23 & 34.8 \\
Primary & 16 & 24.2 \\
Secondary & 11 & 16.7 \\
Higher & & \\
Marital status & 9 & 13.6 \\
Single & 36 & 54.5 \\
Married & 2 & 3 \\
Divorced & 19 & 28.8 \\
Widow & & 100 \\
Dominant side & & \\
Right & & \\
\hline & & \\
\hline
\end{tabular}

Table 2: Time before management and clinical parameters on admission 
Time before consultation in a health facility (hours)

0.5

60

$11(3-24)$

Time before arriving at the reference hospital (hours)

0.5

336

$24(7.3-72)$

Time before doing a CT following arrival at the hospital (hours)

$0 \quad 288 \quad 24(1-35.2)$

\section{Glasgow coma score}

NIHSS score

Systolic blood pressure

Diastolic blood pressure

Temperature

Heart rate

Respiratory rate

Abdominal circumference (cm)
6

3

100

60

36

42

8

73
15

32

260

181

39,3

130

36

132
$15(10.8-15)$

$9(5-12)$

$180(150-210)$

$100(90-120)$

$37.4(37-37.6)$

$80(70-92)$

$20(18-23.3)$

$95(86-104.7)$

Table 3: Stroke topography in our study population 


\begin{tabular}{|c|c|c|c|}
\hline Topography & & Number & Percentage \\
\hline \multicolumn{4}{|l|}{ Cerebral infarction } \\
\hline Anterior cerebral artery & & 2 & 5.7 \\
\hline Middle cerebral artery & & 18 & 51.4 \\
\hline Superficial MCA * & 4 & & 11.4 \\
\hline Deep MCA & 10 & & 28.6 \\
\hline Complete MCA & 4 & & 11.4 \\
\hline Posterior cerebral artery & & 6 & 17.1 \\
\hline Lacunar stroke & & 8 & 22.9 \\
\hline Superficial ACA and MCA** & & 1 & 2.9 \\
\hline \multicolumn{4}{|l|}{ Cerebral hematoma } \\
\hline Deep & & 22 & 71.1 \\
\hline Capsular & & 2 & 6.5 \\
\hline Thalamo-capsular & & 6 & 19.4 \\
\hline Lenticulo-capsular & & 6 & 19.4 \\
\hline Lenticulo-thalamo-capsular & & 8 & 25.8 \\
\hline Lobar & & 6 & 19.4 \\
\hline Brain stem & & 1 & 3.2 \\
\hline Cerebellar & & 2 & 6.5 \\
\hline
\end{tabular}

*MCA: Middle Cerebral Artery; **ACA: Anterior Cerebral Artery

Table IV: Multivariate analysis according to Cox regression model 


\begin{tabular}{lcccc}
\hline Variables & Adjusted Hazard Ratio & 95\% CI & Adjusted p value \\
\hline Dysphagia & 4.21 & 0.77 & 23.23 & 0.1 \\
Detrusor-external sphincter dyssynergia & 1.46 & 0.48 & 4.48 & 0.5 \\
& & & & $0.01 *$ \\
Glasgow coma score & 0.55 & 0.35 & 0.89 & $0.01 *$ \\
Systolic BP & & & & 0.63 \\
Respiratory rate & 1.03 & 1.01 & 1.06 & 0.85 \\
Temperature & 1.04 & 0.88 & 1.23 & 0.12 \\
NIHSS score & & & & \\
\hline
\end{tabular}

Significant association

Figures 


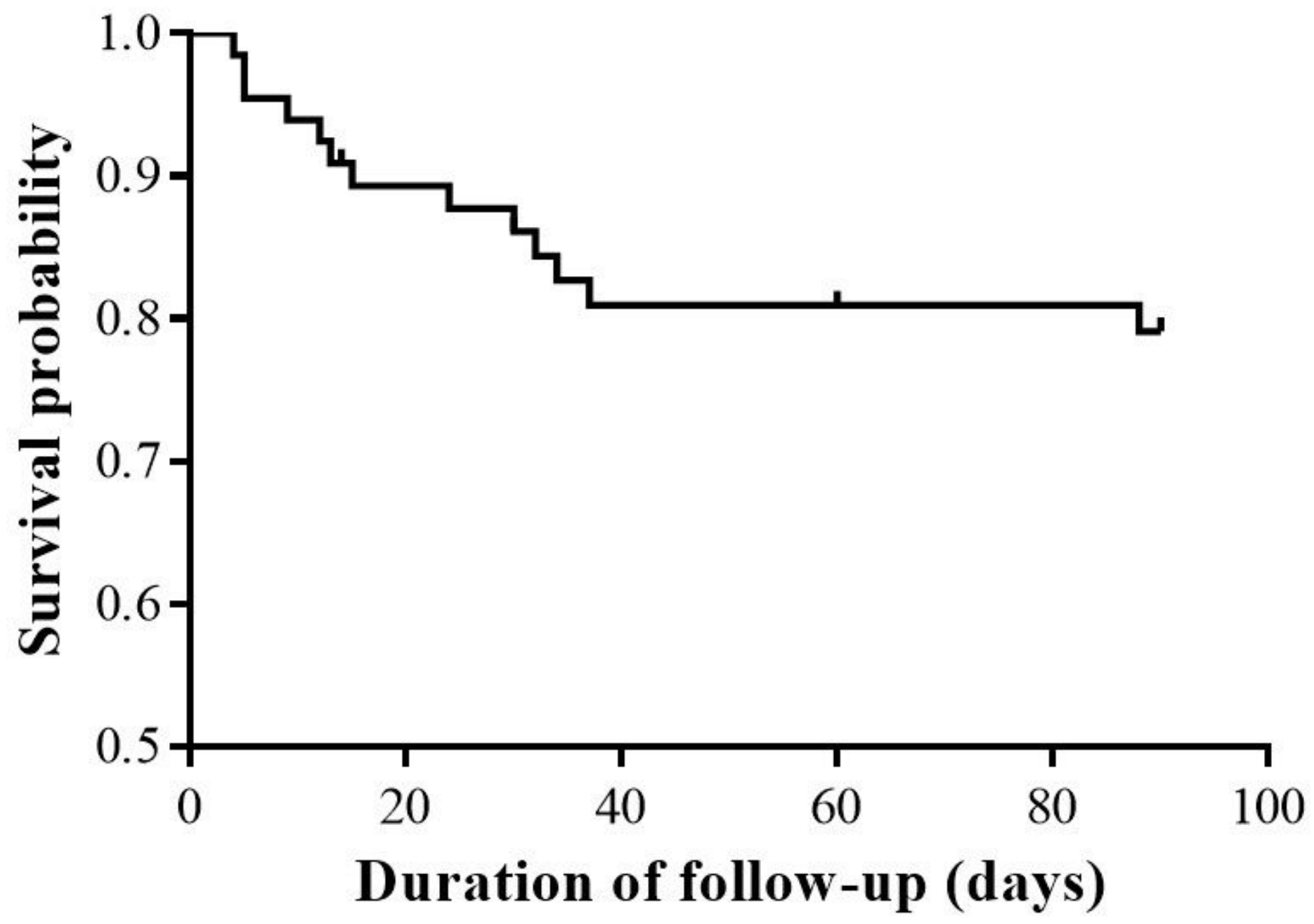

Figure 1

Survival curve of stroke patients on day 90 


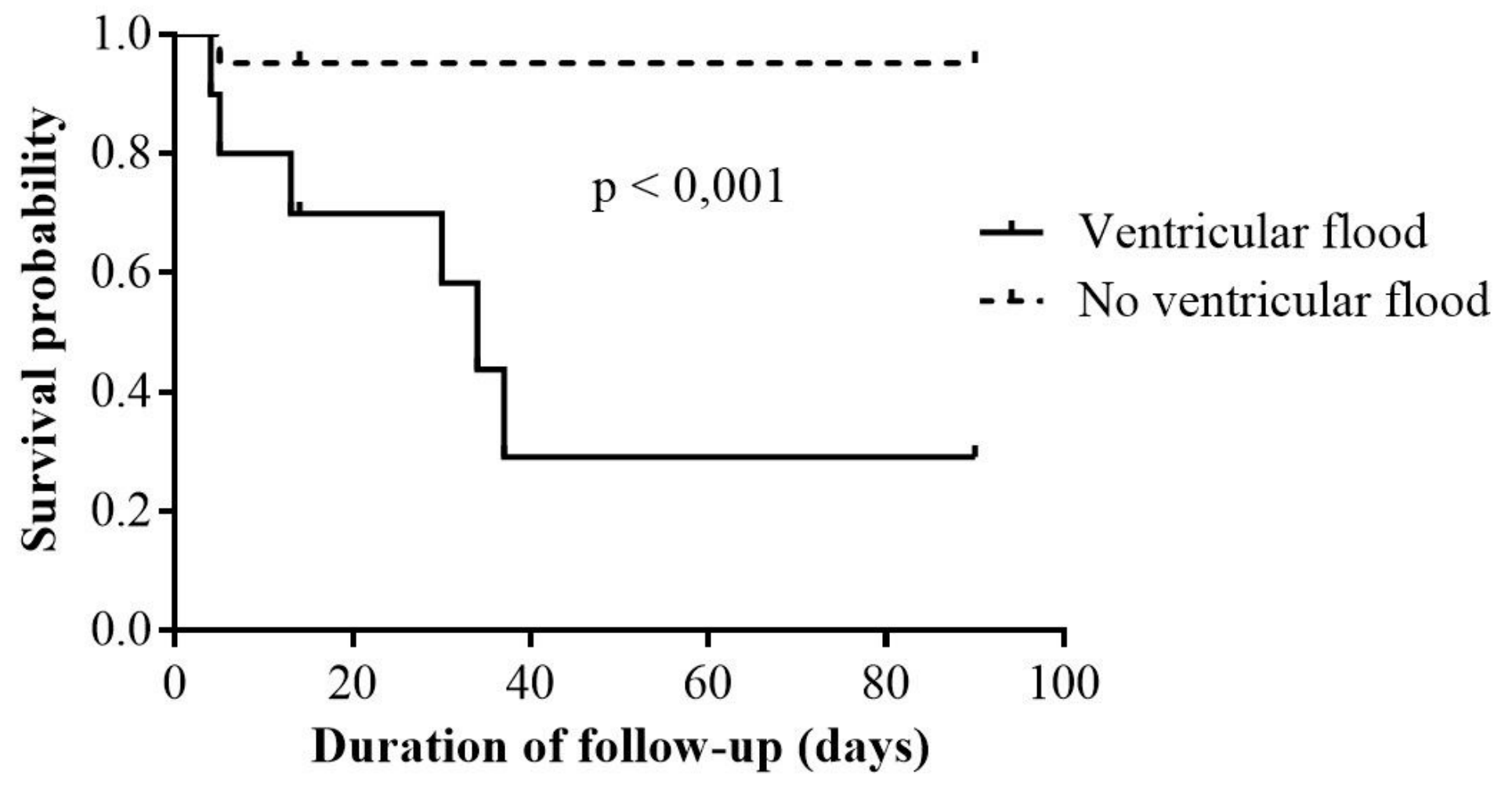

Figure 2

Patients survival curve according to presence or absence of ventricular flood 